\title{
Transfer Learning-based City Similarity Measurement: A Case Study on Urban Hotel
}

\author{
Ganghua Zhang, Xiaoping Che, Shiyao Wei, Tao Na \\ School of Software Engineering \\ Beijing Jiaotong University \\ Beijing, China \\ 19121730@bjtu.edu.cn,xpche@bjtu.edu.cn
}

\begin{abstract}
With the development of modern cities, multiple types and wide distribution of urban data has been gradually collected. Effectively using urban data to solve city development and planning issues has become a research hot-spot. Currently, the data scale in modern cities is quite different, and the fitting degree of machine learning algorithm based on single city is not mature yet. This paper studies the problem with transfer learning technique, and trains the prediction model of urban hotel development scale using multi-source city data. Based on the location data and related information of 15 different cities, the relevant knowledge is transferred, and a city feature extraction and similarity measurement framework is proposed.
\end{abstract}

Index Terms-Transfer Learning, City Similarity, Scale Prediction

\section{INTRODUCTION}

In order to solve the optimal location decision-making problem of enterprises, one of the most direct methods is to recommend the location of enterprise users. In recent years, a large number of academic papers have conducted in-depth research on the location recommendation method [1]-[3]. In the field of urban computing [4], the measurement of urban similarity [5] is one of the key issues. At the same time, the measurement of urban similarity is also the basis of urban transfer learning. At present, many methods of city similarity measurement have been explored. One of the dominant methods is the similarity measurement method [6]-[9] based on different angle of urban feature vector.

In this paper, the distribution and location of urban hotels are taken as the specific research object. The application of transfer learning technology in urban computing is studied experimentally, and an algorithm visualization system is established based on the research results.

The primary contributions are:

- A city similarity measurement algorithm is proposed to measure city similarity in terms of hotel distribution.

- A transfer learning based deep neural network is proposed to increase the prediction accuracy.

- A visualization system of urban hotel development scale prediction is implemented.

\section{RELATED WORK}

The main content of this paper is to solve the problem of urban hotel distribution and location optimization with transfer

\footnotetext{
${ }^{0}$ DOI reference number: 10.18293/SEKE2021-011
}

learning. The related research work can be summarized into three aspects: location recommendation algorithm, city similarity measurement and city measurement based on transfer learning.

\section{A. City similarity measurement}

At present, many methods of city similarity measurement have been explored. One of the dominant methods is the similarity measurement method based on the city feature vectors from different angles. For example, Sheng [10] proposed a geographic similarity region search model based on the approximate search algorithm based on quadtree. In their paper, $\mathrm{K}$ rectangular regions which are most similar to the target query area are found. The regional similarity evaluation method and feature extraction method proposed in their paper have important reference significance for the city similarity evaluation algorithm. Diserud [11] proposed a multi site similarity measurement method in the biological field. The measurement method uses the species information shared by more than two sites, and avoids the covariance problem between pairwise similarity in multi-site research.

\section{B. Urban computing based on Transfer Learning}

Based on the definition of transfer learning, many papers have studied the application of transfer learning technology in the field of urban computing. Wang discussed the general process of urban transfer learning in Smart City Development With Urban Transfer Learning [12], and provided guidance for urban planners and relevant practitioners on how to apply this novel learning method.

This paper also puts forward the general steps to be followed in the application of transfer learning in urban planning, as well as case studies on public safety and traffic management, and summarizes some possible research directions.

Based on the urban transfer learning guide, a variety of transfer learning models for urban data have been proposed and optimized. For example, Yao [13] proposed multi-source knowledge transfer frameworks of MultiSourceTrAdaBoost and TaskTrAdaBoost, which can effectively solve the problem of negative transfer [14] in urban data transfer learning, and can be applied to class identification and specific object detection of migrated objects. Tan proposed a multi-source transfer algorithm [15], which can make different views from different 
sources and sources complement each other through collaborative training framework, so as to jointly utilize knowledge from different sources and views, and modify the distribution differences in different fields.

\section{The shortcomings of existing research}

There are some shortcomings in the two types of city similarity research directions introduced in this section. The first type of similarity research direction based on city feature vector still has the problems of optimizing the extraction method of feature vector and the selection of distance measurement method when measuring the city similarity. The problem of second type is that the standard of city score is not universal.

\section{CITY SIMILARITY MEASUREMENT ALGORITHM}

The similarity measurement between cities can be carried out for different data types in cities.

\section{A. Urban similarity computing algorithm architecture}

This section introduces the city similarity measurement algorithm proposed in this paper from the overall architecture level.

1) Basic concepts: Before introducing the architecture of city similarity algorithm, several basic concepts need to be defined clearly.

a) City grid: In order to extract the features of cities and analyze the regional data better, we put forward the concept of urban gridding.

The number of POI grid attributes can be defined as the variable shown in formula (1)

$$
\operatorname{Prop}{ }_{g(i, j)}^{A}
$$

Where a represents a specific attribute and $g(i, j)$ represents the coordinates of the lattice.

b) City similarity: The city similarity measurement defined in this paper comes from the perspective of research objectives, which takes the feature dimensions to be studied and the related dimensions with strong correlation as the feature space of the experiment, which can solve the problem of difficult data acquisition and minimize the impact of redundant features on the experimental results.

2) Algorithm architecture: After defining the above basic concepts, the overall architecture of the city similarity measurement algorithm is shown in Fig.1.

The similarity measurement part is the core part of the algorithm mainly measures the similarity between the macro feature vectors of two target cities. This algorithm uses Euclidean distance to calculate the distance between the two macro feature vectors, and takes the measurement results as a part of the final similarity results.

\section{B. City similarity measurement}

This section introduces the city similarity measurement algorithm in detail from the data preparation, feature extraction and algorithm construction, which is the basis and premise of building the transfer learning model introduced in the next Chapter.

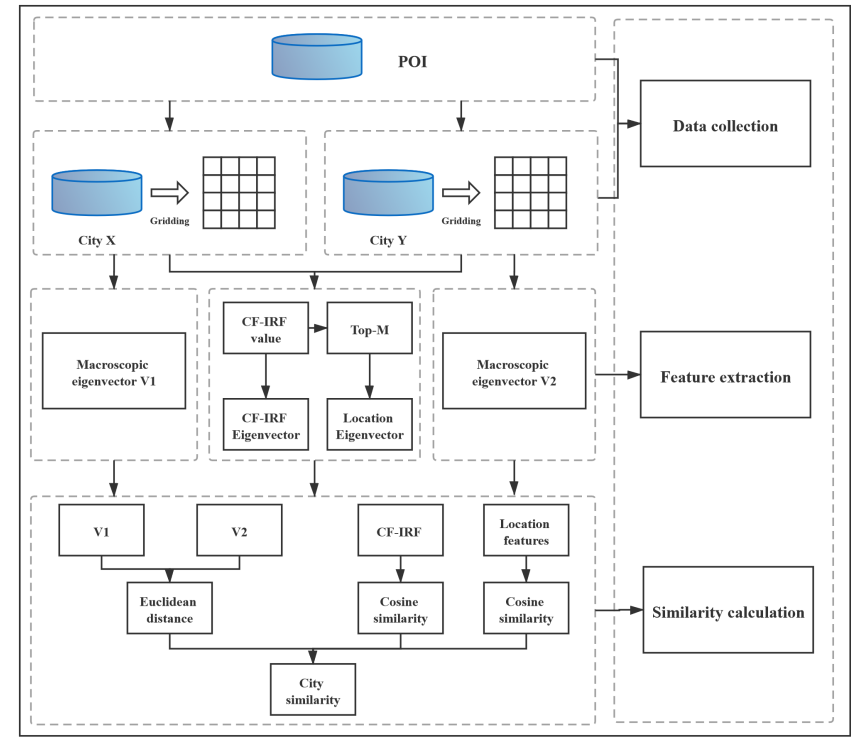

Fig. 1. Urban similarity measurement algorithm architecture

1) Data preprocessing: The grid POI data of 15 different types of cities are collected as the data sets of this paper, which are Seoul, Tokyo, Beijing, Shanghai, Chongqing.etc. After finding the maximum and minimum longitude and latitude range of each city's City Hotel, the grid division is carried out. The part of specific collection and division results are as follows.

TABLE I

City DATA TABLE 1

\begin{tabular}{cccccc}
\hline \hline City & Beijing & Seoul & Tokyo & Shanghai & Dalian \\
\hline \hline POI & 635096 & 211089 & 391174 & 617870 & 119411 \\
Grid & 957 & 142 & 465 & 667 & 195 \\
\hline
\end{tabular}

2) Feature extraction: The definition variable a represents the target prediction characteristics, the variable $\mathrm{X}$ represents the target research city, the variable $R$ represents the grid row number of the city divided by the target research city $\mathrm{x}$, and the variable $\mathrm{C}$ represents the column number of the city grid:

$$
N u m_{\text {grids }}^{X}=r * c
$$

Equation (3) represents the total number of grids in the target study city $\mathrm{X}$. Then there are:

$$
\operatorname{Sum}_{A}^{X}=\sum_{i=0, j=0}^{i=r, j=c} \operatorname{Prop}_{g(i, j)}^{A}
$$

Equation represents the total number of feature $\mathrm{a}$ in the target city. After defining the basic variables that need to be used in the macro feature vector, the feature vector $\mathrm{V}$ of the macro part of city $\mathrm{x}$ is defined $V_{m}^{X}$ define a $\mathrm{S}$ is the 19 feature dimensions of urban grid, where a is the target feature, B is the target feature $S$ represents the rest of the features, and the 
feature vector is defined as 18 dimensions, one of which can be defined as formula (4).

$$
f(\theta)_{X}=\frac{\sum_{i=0, j=0}^{r, c} \frac{\operatorname{Prop}_{g(i, j)}^{\theta} * \operatorname{Sum}_{A}^{X}}{\left(\sum_{p=B}^{S} \operatorname{Prop}_{g(i, j)}^{P}\right) * \operatorname{Prop}_{g(i, j)}^{A}}}{N u m_{\text {grids }}^{X}} \theta \in[B, S]
$$

3) City similarity measurement: The city similarity measurement algorithm proposed in this paper measures the similarity of the target city from two levels, namely, the whole city level and the single grid level. Among them, the overall level similarity measurement mainly uses the city macro feature vector extraction method introduced before. The measurement formula of the overall level similarity of city $\mathrm{X}$ and city $\mathrm{Y}$ is shown in formula (5).

$$
\operatorname{Sim}_{X}^{Y}=\frac{1}{10000 * \sqrt{\sum_{i=0}^{17}\left(V_{X}[i]-V_{Y}[i]\right)^{2}}}
$$

The detailed measurement process is shown by the pseudo code of algorithm 1 below.

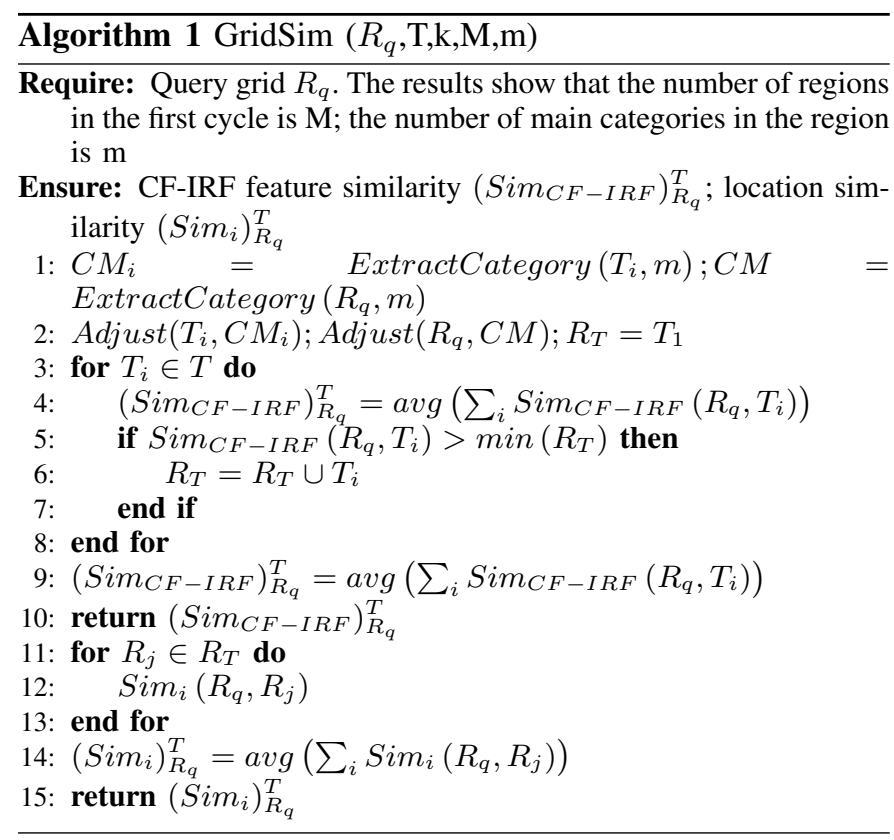

\section{PREDiction ALGORITHM OF URBAN HOTEL} DEVELOPMENT SCALE BASED ON TRANSFER LEARNING

\section{A. Prediction algorithm of urban hotel development scale}

This section will introduce the flow and principle of the algorithm in detail from three parts: algorithm architecture, neural network structure and algorithm optimization process.

1) Algorithm architecture: In this paper, the urban hotel development trend prediction problem is defined as a classification problem. The category labels to be predicted represent the high, medium and low number of urban hotels in the target grid.

As shown in Fig. 1 described before, the similarity between the target city and other cities is calculated. The city data with the highest similarity and the target city data are input into the neural network as the training set for iteration. Then, the normal Kaiming initialization method is used to set the initialization parameters of the neural network. The parameter optimization algorithm in the iteration process is Adam, and finally the model is compared on the test set The performance of the model was evaluated. The training process of the model is shown in algorithm 2 .

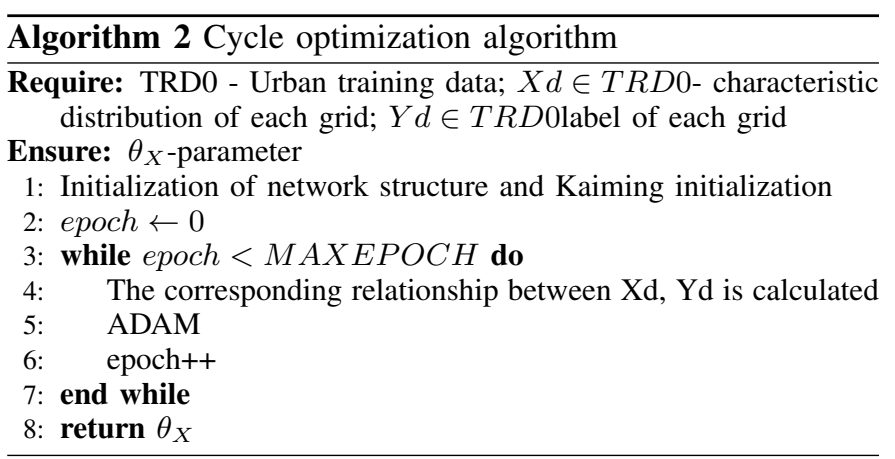

\section{B. Experimental results}

This section introduces the whole process of model training and the final experimental setup and results.

Beijing is selected as the target experimental city. Firstly, the similarity between the other 14 cities in the dataset and Beijing is calculated according to the city similarity measurement method described in Chapter 3. The distribution of the measurement results is shown in Fig.2(a).

The experimental results of target cities show that the higher the similarity with the target city, the better the learning effect is. Therefore, the city similarity measurement algorithm proposed in this paper can correctly measure the similarity degree of POI distribution between cities, and then transfer learning based on the similarity to get the prediction model of City Hotel development trend The accuracy has been improved.

\section{Algorithm visualization}

Based on the prediction model of urban hotel development scale based on transfer learning, this section visualizes the established algorithm model and applies the algorithm model to practical application, which verifies the effectiveness of the model in practical application. The screen shots of running results are shown in Fig. 4.

\section{Summary and Prospect}

According to the given direction of the experiment, we design and implement the city POI feature extraction methods of macro level and grid level. With the distance measurement method of Euclidean distance and cosine similarity, we finally realize the city similarity measurement algorithm. Using this algorithm.

Based on the city similarity measurement algorithm, this paper introduces the transfer learning based deep neural network method, designs and implements the benchmark experiment. 


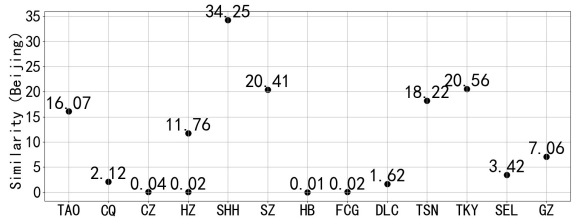

(a) Beijing

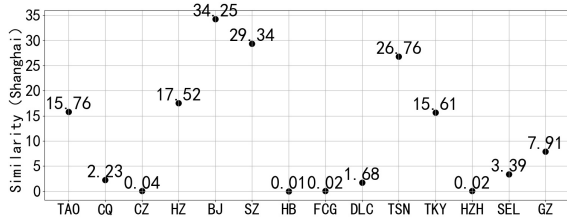

(b) Shanghai

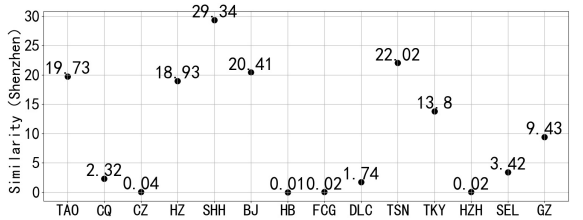

(c) Shenzhen

Fig. 2. Similarity distribution of Beijing, Shanghai and Shenzhen

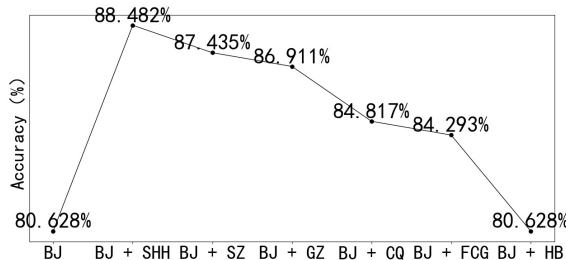

(a) Beijing

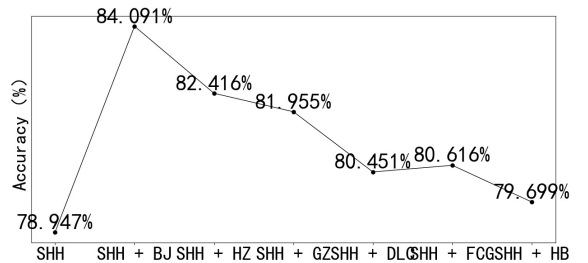

(b) Shanghai

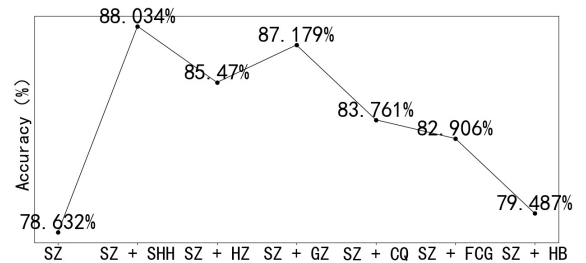

(c) Shenzhen

Fig. 3. Prediction Accuracy of Beijing,Shanghai and Shenzhen

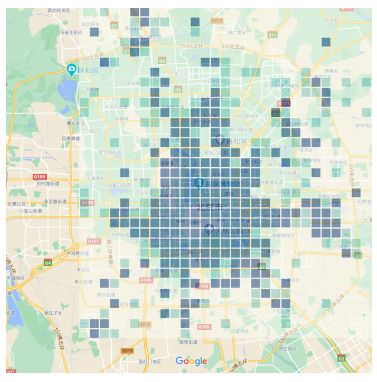

(a) Beijing

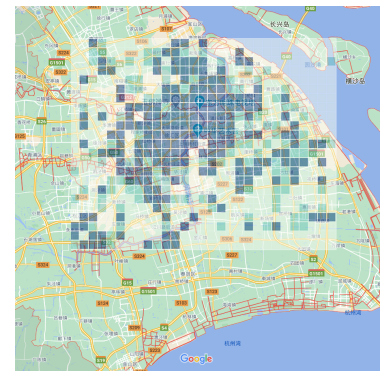

(b) Shanghai

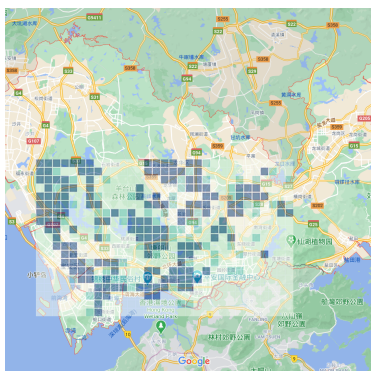

(c) Shenzhen

Fig. 4. Beijing, Shanghai and Shenzhen Prediction Map

At the same time, according to the results of city similarity distribution, six representative city data are selected to train the transfer learning model. The experimental results show that the urban data transfer learning effect and source The results also verify the correctness and effectiveness of the proposed method.Finally,a visualization system of urban hotel development trend prediction algorithm is designed and implemented.

\section{ACKNOWLEDGMENT}

Thanks for the guidance of associate professor XiaopingChe, School of software, Beijing Jiaotong University, and the help of Tao-Na and Shiyao-Wei in the experiment.

\section{REFERENCES}

[1] Y. Li, Y. Zheng, S. Ji, W. Wang, Z. Gong et al., "Location selection for ambulance stations: a data-driven approach," in Proceedings of the 23rd SIGSPATIAL International Conference on Advances in Geographic Information Systems. ACM, 2015, p. 85.

[2] C. Yin, S. Ding, and J. Wang, "Mobile marketing recommendation method based on user location feedback," 2019.

[3] Y. Zheng, L. Capra, O. Wolfson, and H. Yang, "Urban computing: concepts, methodologies, and applications," ACM Transactions on Intelligent Systems and Technology (TIST), vol. 5, no. 3, p. 38, 2014.

[4] Shklovski, Irina, Chang, Michele, and F., "Urban computing: Navigating space and context." Computer, 2006.
[5] G. Q. Li and S. J. Liu, "The calculation method of similarity degree for city logistics facilities," Advanced Materials Research, vol. 219-220, pp. 1621-1624, 2011.

[6] MOVE'19: Proceedings of the 1st ACM SIGSPATIAL International Workshop on Computing with Multifaceted Movement Data. New York, NY, USA: Association for Computing Machinery, 2019.

[7] Linden, G., Smith, B., York, and J., "Amazon.com recommendations: item-to-item collaborative filtering," Internet Computing, IEEE, vol. 7, no. 1, pp. 76-80, 2003.

[8] J. Herlocker, J. Konstan, and J. Riedl, "Explaining collaborative filtering recommendations," Proceedings of the ACM Conference on Computer Supported Cooperative Work, 012001.

[9] L. Deng and D. Yu, "Deep learning: Methods and applications," Foundations Trends in Signal Processing, vol. 7, no. 3, 2014.

[10] C. Sheng, Y. Zheng, W. Hsu, M. L. Lee, and X. Xie, "Answering top-k similar region queries," in International Conference on Database Systems for Advanced Applications. Springer, 2010, pp. 186-201.

[11] O. H. Diserud and F. Ødegaard, "A multiple-site similarity measure," Biology letters, vol. 3, no. 1, pp. 20-22, 2007.

[12] L. Wang, B. Guo, and Q. Yang, "Smart city development with urban transfer learning," Computer, vol. 51, no. 12, pp. 32-41, 2018.

[13] Y. Yao and G. Doretto, "Boosting for transfer learning with multiple sources," in 2010 IEEE Computer Society Conference on Computer Vision and Pattern Recognition. IEEE, 2010, pp. 1855-1862.

[14] K. Weiss, T. M. Khoshgoftaar, and D. Wang, "A survey of transfer learning," Journal of Big data, vol. 3, no. 1, p. 9, 2016.

[15] B. Tan, E. Zhong, E. W. Xiang, and Q. Yang, "Multi-transfer: Transfer learning with multiple views and multiple sources," in Proceedings of the 2013 SIAM International Conference on Data Mining. SIAM, 2013, pp. 243-251. 